\title{
Gpr97 is essential for the follicular versus marginal zone B-lymphocyte fate decision
}

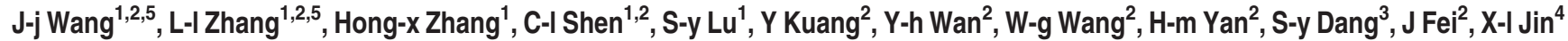 \\ and Z-g Wang ${ }^{*, 1,2,3}$
}

Gpr97 is an orphan adhesion GPCR and is highly conserved among species. Up to now, its physiological function remains largely unknown. Here, we show that Gpr97 deficiency results in an extensive reduction in B220 ${ }^{+}$lymphocytes in mice. More intensive analyses reveal an expanded marginal zone but a decreased follicular B-cell population in $G$ pr97 ${ }^{-/-}$spleen, which displays disorganized architecture characterized by diffuse, irregular B-cell areas and the absence of discrete perifollicular marginal and mantle zones. In vivo functional studies reveal that the mutant mice could generate antibody responses to T cell-dependent and independent antigens, albeit enhanced response to the former and weakened response to the latter. By screening for the molecular events involved in the observed phenotypes, we found that lambda 5 expression is downregulated and its upstream inhibitor Aiolos is increased in the spleen of mutant mice, accompanied by significantly enhanced phosphorylation and nuclear translocation of cAMP response element-binding protein. Interestingly, increased constitutive $\mathrm{Nf}-\boldsymbol{\kappa} \mathrm{b}$ p50/p65 expression and activity were observed in $\mathrm{Gpr} \mathrm{7}^{-/-}$spleen, implicating a crucial role of Gpr97 in regulating Nf- $\kappa \mathrm{b}$ activity. These findings uncover a novel biological function of Gpr97 in regulating B-cell development, implying Gpr97 as a potential therapeutic target for treatment of immunological disorders.

Cell Death and Disease (2013) 4, e853; doi:10.1038/cddis.2013.346; published online 10 October 2013

Subject Category: Immunity

B cells develop from hematopoietic stem cells in the BM. Early development and commitment to the B-cell lineage depend on diverse transcription factors, including early B-cell factor, PU.1, E2A and PAX5. ${ }^{1}$ The earliest committed B-cell progenitors in the active cell cycle begin DJ rearrangement, followed by $V(D) J$ rearrangement at the $\mathrm{H}$-chain loci at the pro-B stage. After a productive $\mathrm{V}(\mathrm{D}) \mathrm{J}$ recombination, the cytoplasmic $\mu$ heavy chains (c $\mu \mathrm{HCs}$ ) are expressed and then paired with surrogate $L$ chains (VpreB and $\lambda 5$ proteins) to form the pre-B-cell receptor (BCR) at the pre-B-cell stage. Expression of the pre-BCR on the cell surface allows the clonal expansion and rearrangement of immunoglobulin-light chain $(I g L)$ gene segments. ${ }^{2}$ The presence of surface IgM permits negative selection to occur (in which tolerance mechanisms delete, anergize or edit autoreactive clones). ${ }^{3}$ Naive $\mathrm{B}^{2} 20^{+} \operatorname{lgM}^{+} \mathrm{B}$ cells that survive negative selection then exit the marrow.

B cells continue their development in the spleen, where they pass through transitional stages transitional type 1 (T1) and transitional type 2 (T2) and are subjected to a further round of negative selection before becoming totally mature. ${ }^{4}$ The most prominent mature $\mathrm{B}$ cells in the spleen are follicular (FO)
B cells, which continue circulating to the follicles in the spleen, to the lymph nodes and to the BM until they either die or encounter cognate antigen and undergo further maturation. Marginal zone (MZ) B cells, located at the outer rim of follicles, represent a smaller fraction of splenic $B$ cells. ${ }^{5}$ The $M Z$ and FO B-cell subsets differ significantly in phenotype, function and anatomical location. How these subpopulations are selected remains incompletely understood. Growing evidence has supported an important role for transcription factors in regulating $\mathrm{MZ}$ and $\mathrm{FO} B$-cell fate. This is largely based on the observations that genetically mutant mice deficient in a series of transcription factors exhibited imbalanced development of $\mathrm{MZ}$ and FO B cells. ${ }^{6-11}$

The adhesion GPCRs, which have 33 members in humans, contain large extracellular $\mathrm{N}$-terminal domain containing a range of protein domains found in cell adhesion proteins, and C-terminal domain homologous to secretin-like GPCR. ${ }^{12-14}$ $\mathrm{N}$ - and $\mathrm{C}$-terminal domains can be autocatalytically cleaved at the membrane-proximal GPCR proteolytic site domain, which is a characteristic feature of this receptor family. ${ }^{15}$ The majority of adhesion GPCRs are still orphans, for which neither ligand nor function is known. There is increasing

\footnotetext{
${ }^{1}$ State Key Laboratory of Medical Genomics, Research Center for Experimental Medicine, Rui-Jin Hospital affiliated to Shanghai Jiao Tong University School of Medicine (SJTUSM), Shanghai, China; ${ }^{2}$ Shanghai Research Center for Model Organisms, Shanghai, China; ${ }^{3}$ Model Organism Division, Department of Medical Genetics, E-Institutes of Shanghai Universities, SJTUSM, Shanghai, China and ${ }^{4}$ Department of Pathology, Shanghai Rui-Jin Hospital affiliated to SJTUSM, Shanghai, China ${ }^{*}$ Corresponding author: Z-g Wang, State Key Laboratory of Medical Genomics, Research Center for Experimental Medicine, Rui-Jin Hospital affiliated to Shanghai Jiao Tong University School of Medicine (SJTUSM), 197 Rui Jin Road II, Building \#23, Shanghai 200025, China. Tel: +86 21 64457997; Fax: +86 21 64457997; E-mail: zhugangw@shsmu.edu.cn

${ }^{5}$ These authors contributed equally to this work.

Keywords: Gpr97; knockout mice; B lymphopoiesis; follicular B cells; lambda 5 gene

Abbreviations: GPCR, G protein-coupled receptor; FO, follicular; MZ, marginal zone; BM, bone marrow; BMD, bone mineral density; PB, peripheral blood; GC, germinal center; PNA, peanut agglutinin; CFA, complete Freund's adjuvant; IFA, incomplete Freund's adjuvant

Received 26.4.13; revised 23.7.13; accepted 29.7.13; Edited by Y Shi
} 
evidence for the roles of adhesion GPCRs in the central nervous system, immune system and tumorigenesis. For example, CELSR $1-3^{16,17}$ and latrophilin $1^{18}$ coordinate neuronal development and neurotransmitter release, respectively. CD97 $7^{19,20}$ and EMR1-3 ${ }^{21-23}$ are involved in coordinating both the innate and the acquired immune responses. GPR124 promotes tumor angiogenesis. ${ }^{24}$ Although there is no consensus yet about the physiological functions of adhesion GPCRs and their molecular mechanisms of signaling, the existing data suggest that this receptor class mediates essential cell-cell and cell-matrix interactions. $^{12,25}$

GPR97 is an orphan adhesion GPCR with homology to the better characterized HE6 (human epididymis-specific protein 6) and GPR56 (human brain-specific protein). Like HE6 and GPR56, GPR97 possesses both an exceptionally long extracellular region, characteristic of cell adhesion proteins and an intracellular region reminiscent of other GPCRs. ${ }^{26,27}$ GPR97 was found by searching human genome databases. ${ }^{26}$ Previous work revealed that Gpr97mRNA is highly expressed in immune cells. ${ }^{28}$ Other study has shown that GPR97 is coupled to $G_{0}$, which means that the inactivation of GPR97 would lead to an increase in cAMP levels in target cells. ${ }^{29}$ To investigate the biological function of Gpr97 in vivo, we have generated $G$ pr97 ${ }^{-/-}$mice. The phenotypic analyses have demonstrated an indispensable role of Gpr97 in maintaining B-lymphocyte population, especially in regulating constitutive CREB and Nf- $\kappa$ b activities.

\section{Results}

Expression profile of Gpr97 in mice. To determine the expression pattern of Gpr97 in normal adult mice, we performed semi-quantitative and real-time reverse transcription (RT)-PCR analyses on various mouse tissues. As shown in Supplementary Figure S1, the highest expression level of Gpr97 mRNA was found in BM, and relatively low but detectable expression levels were also observed in heart, kidney and spleen tissues, implicating the tissue compartments where Gpr97 could execute its physiological functions in mice.

Gpr97 $^{-1-}$ mice are born alive and appear grossly normal. To explore the physiological function of Gpr97 in vivo, we generated a mouse model with global-targeted deletion of Gpr97 (Figure 1a). The homologous recombination in ES cells was confirmed (Figure 1b), and the genotypes of mice were verified by PCR analysis of genomic DNA (Figure 1c). The absence of Gpr97 expression was confirmed by analyzing the Gpr97 mRNA by RT-PCR (Figure 1d) and protein expression by western blot analysis (Figure 1e). As expected, Gpr97 mRNA and protein were disrupted in the

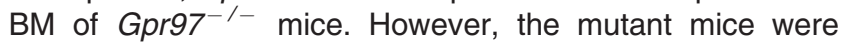
viable and reached the adult stage without any gross developmental abnormalities, suggesting that Gpr97 is not indispensible for normal development.

Reduction of B-cell population in $G p r 97^{-1-}$ mice. Considering the features of Gpr97 expression profile in mice,

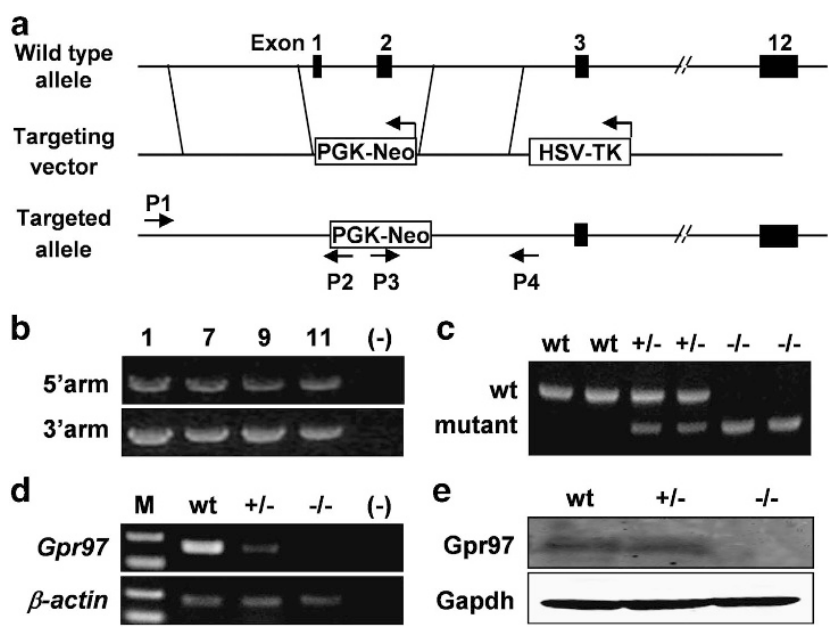

Figure 1 Targeted disruption of Gpr97 in mice. (a) Schematic representation of Gpr97 gene KO strategy. The targeting vector was designed to delete exon 1 harboring ATG codon and exon 2. Genomic regions amplified by PCR for genotyping are indicated by arrows. Exons are indicated as black boxes. (b) PCR analysis of ES cell clones. Genomic DNA extracted from ES clones was amplified using $5^{\prime}$-external and $3^{\prime}$-external primers as shown in panel (a). Homologous recombination events yielded a $6.5-\mathrm{kb}$ or $3.8-\mathrm{kb}$ fragment, respectively. (c) PCR genotyping of progenies from heterozygous matings. WT $(+/+)$, heterozygous $(+/-)$ and homozygous $(-/-)$ mice were identified by PCR amplification of the fragments specific for either Gpr97 WT allele (944-bp) or the mutant allele (730-bp). (d) RT-PCR analysis for Gpr97 mRNA in BM of WT, Gpr97 ${ }^{+1-}$ and $\mathrm{Gpr}^{-1} 7^{-1}$ mice. The Gpr97-specific 192-bp product is absent in $\mathrm{Gpr}^{-1-}$ and reduced in $\mathrm{Gpr} 7^{+1-}$ mice. $\beta$-actin was used as a loading control. (e) Immunoblotting of BM protein samples with antibodies against Gpr97 and Gapdh shows absence of Gpr97 in mutant mice

we performed several phenotypic screening tests for detection of some parameters potentially affected due to Gpr97 deficiency. It was found that no significant differences in body weight, ratios of thymus or spleen weight to body weight, as well as bone mineral density between sex- and age-matched WT and $\mathrm{Gpr}^{-1-}$ mice (Supplementary Figure S2a-d). Hematological examination revealed that the numbers of white blood cells, red blood cells and platelets remained unaffected in PB of $\mathrm{GprO7}^{-/-}$mice (Supplementary Figure S2e). The differential counting of BM cells was performed by flow cytometry using lineage-specific cellsurface markers. The results showed that the percentages of $\mathrm{CD}^{+}, \mathrm{Ter} 119^{+}, \mathrm{CD}_{4} 1^{+}$and $\mathrm{Gr}-1^{+}$cells were comparable

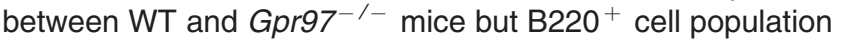
was decreased in Gpr97-deficient BM cells (Supplementary Figure S2f). More intensive analysis revealed that the reduction of $\mathrm{B}^{2} 20^{+}$cells was observed not only in BM but also in spleen and PB of the mice lacking Gpr97 as compared with WT littermates (Figure 2a). The percentages of $T$ cells and granulocytes were not significantly different between WT and Gpr97 ${ }^{-/-}$mice as judged by CD3 or Gr-1 staining, respectively (Figure $2 \mathrm{~b}$ ). These results suggested that Gpr97 is essential in maintaining B220 ${ }^{+}$-cell population. To address whether the reduction of $B$ lymphocytes was due to impaired cell proliferation or increased apoptosis, singlecell suspensions of splenocytes were cultured with or without LPS $(20 \mu \mathrm{g} / \mathrm{ml})$ stimulation. MTT assay revealed that 
a

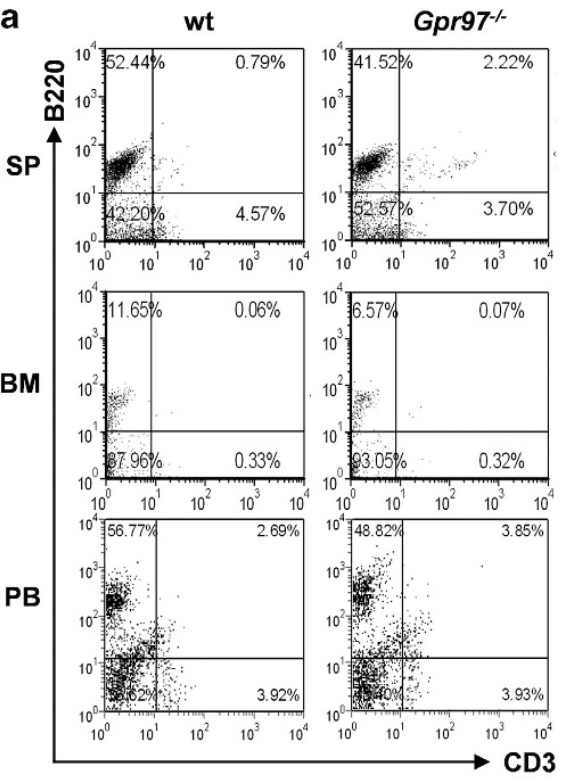

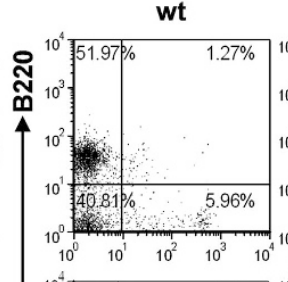
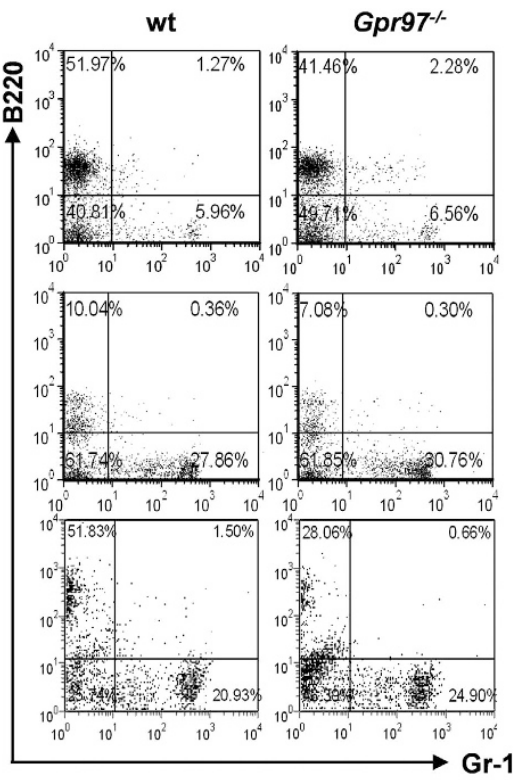

b
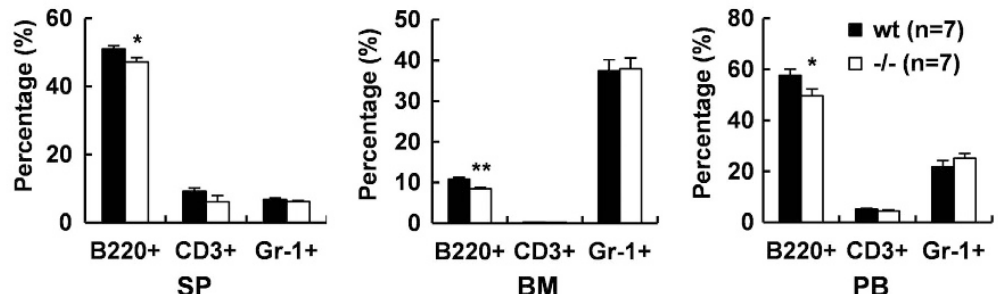

Figure 2 Absence of Gpr97 impairs B lymphopoiesis in BM, spleen (SP) and peripheral blood (PB). (a) Splenocytes, BM and PB cells were recovered from 12-week-old WT and age-, sex-matched KO mice ( $n=7$ for each). The cells were labeled with monoclonal antibodies as indicated and subjected to flow cytometric analysis. The numbers in each quadrant indicate the percentages of cell populations. (b) The relative numbers of $\mathrm{B}_{22}{ }^{+}, \mathrm{CD}^{+}$and $\mathrm{Gr}-1^{+}$cells were expressed as mean $\pm \mathrm{S}$.E.M. $(n=7)$. Individual fractions defined in (a) were calculated. ${ }^{\star} P<0.05$; ${ }^{\star} P<0.01$

Gpr97 ${ }^{-/-}$splenocytes proliferated as rapidly as WT cells upon LPS treatment (data not shown). However, annexin V/PI staining and FACS analyses showed that $\mathrm{B}^{2} 20^{+}$cells deficient for Gpr97 had increased apoptosis when compared with WT cells cultured for $24 \mathrm{~h}$ in the presence or absence of LPS (Supplementary Figure S3). We also analyzed $\mathrm{Bcl}-2$, Bax and caspase-3 mRNA levels, as well as caspase-3 activity in splenic $\mathrm{B}_{2} 2 \mathrm{O}^{+}$cells, and found that $\mathrm{Bcl}-2$ expression level is decreased and caspase- 3 activity is increased in Gpr97 $^{-/}$splenic B lymphocytes cultured for $24 \mathrm{~h}$ with or without LPS (Supplementary Figure S4). Taken together, these observations demonstrate that the increased apoptosis of $\mathrm{B}^{2} 20^{+}$cells in $\mathrm{Gpr} 97^{-/-}$spleen was attributed to enhanced caspase-3 activity.

Abnormal B-cell subpopulations in BM and spleen of $\mathrm{Gpr}^{-1 /}$ mice. To further characterize the reduction of B-cell population in Gpr97-deficient mice, we performed an extensive survey of surface marker expression on B-lineage cells in BM. ${ }^{30,31}$ It was found that mature $\mathrm{B}\left(\mathrm{B}^{2} 20^{+} \mathrm{IgM}^{+}\right.$ $\operatorname{lgD}{ }^{+}, \mathrm{M}$ )-cell populations were reduced in $\mathrm{Gpr}^{-1-}$ mice as compared with WT controls. The percentages and absolute cell numbers of mature $B$ cells were reduced by $32.7 \%$ and $36.2 \%$, respectively. Although pro-B (CD43 ${ }^{+}$
$\left.B 220^{\text {int }}\right)$, pre-B/immature $B\left(\mathrm{CD} 43^{-} \mathrm{B} 220^{\text {int }}\right)$ and immature $\mathrm{B}$ $\left(\mathrm{B}^{2} 20^{+} \mathrm{IgM}^{+} \mathrm{IgD}^{-}\right.$, IM) cell populations in Gpr97 mutant BM were comparable to those found in WT littermates (Figures $3 a$ and $b$ ). These findings suggest that Gpr97 is not involved in early B-lineage cell commitment but is required for development of mature $B$ cells in secondary lymphoid tissues.

By analyzing the splenic B-cell compartment, we observed that the percentages of mature $\mathrm{B}\left(\mathrm{B} 220^{+} \mathrm{CD} 21^{\text {int }}\right.$ IgM $\left.{ }^{\text {int }}\right)$ and

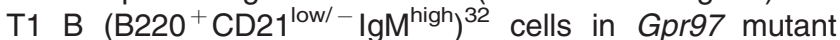
spleens were lower than those in WT controls (Figure 3c, top; 3d, top left). Accordingly, the absolute numbers of mature $B$ and $T 1 B$ cells were significantly reduced due to a decrease in total splenic B cells in mutant mice as compared with WT controls (Figure 3d, top right). Meanwhile, the T2 B (B220 ${ }^{+}$ $\left.\mathrm{CD} 21^{\text {high }} \operatorname{lgM}^{\text {high }}\right)^{33}$ cells of mutant mice were found significantly increased with regard to percentages, but no significant changes were observed in the absolute cell numbers (Figure 3c, top; 3d, top right). By means of flow cytometry, mature $B$ cells in spleen were separated into $M Z B$ and FO B cells in terms of the surface expression of CD21 and $\mathrm{CD}_{23} 3^{34,35}$ (Figure $3 \mathrm{c}$, bottom). Our results revealed that $\mathrm{MZ}$ $\mathrm{B}$ cells $\left(\mathrm{B} 220^{+} \mathrm{CD} 21^{\text {high }} \mathrm{CD} 23^{\text {low } /-}\right)$ were markedly increased by 3.8 -fold in percentage and 2.5 -fold in absolute numbers 
a
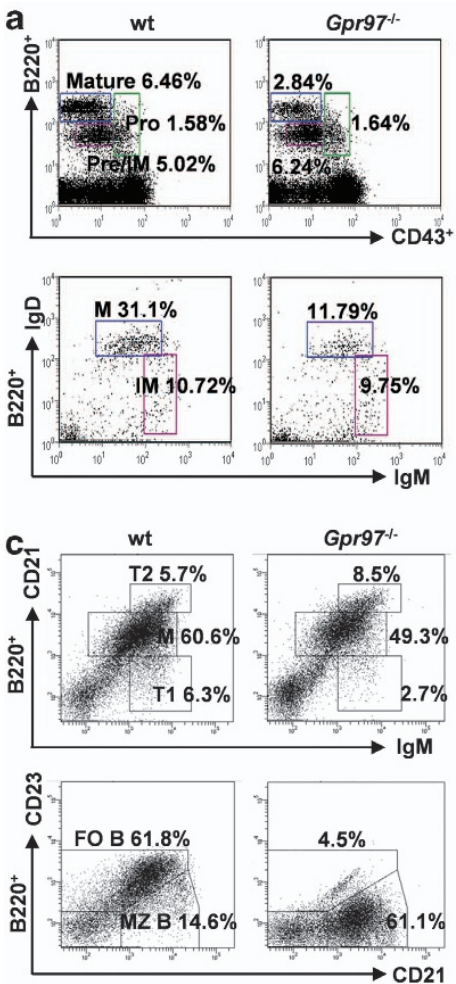
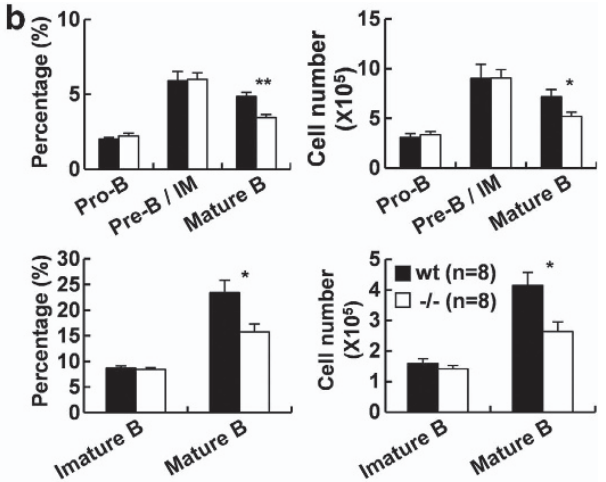

d
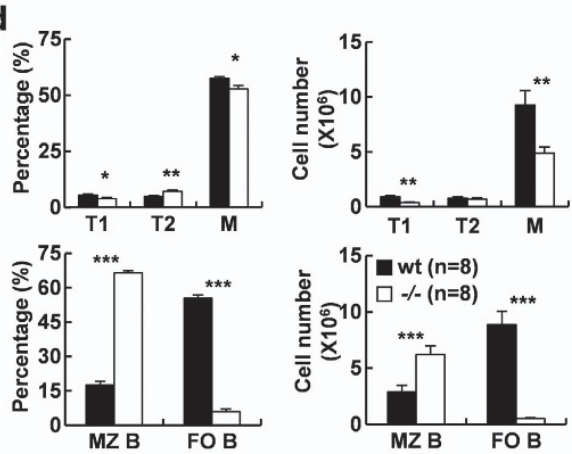

Figure 3 Analysis of subpopulations of B cells from WT and KO mice. (a) BM cells from WT and Gpr97-KO mice ( $n=8$ for each) were labeled for B220 and CD43 (upper panels), or B220, IgM, IgD (lower panels), and gated on total lymphocytes or B220 ${ }^{+}$cells, respectively. Numbers represent the percentages of cells in the plot that fall within the different regions, corresponding to different stages of B-cell differentiation. (b) The relative and absolute numbers of $C D 43^{+} B 220^{\text {int }}($ pro-B), CD43- B220 int $($ pre-B/

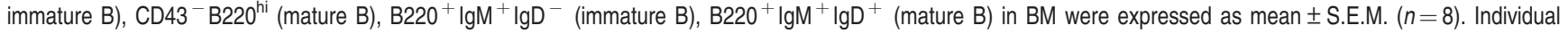
fractions defined in (a) were calculated. (c) Flow cytometry profiles of the spleen of WT and KO mice labeled for B220, IgM, CD21 (upper panels), or B220, CD21, CD23 (lower panels), and gated on $\mathrm{B}_{22} \mathrm{O}^{+}$cells. Numbers represent the percentages of cells in the plot that fall within the different regions, corresponding to different stages of $\mathrm{B}$ cell

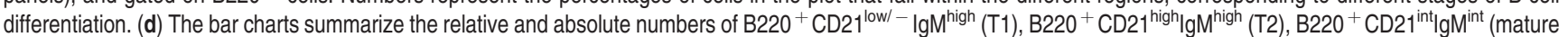
$\mathrm{B}, \mathrm{M}), \mathrm{B} 22 \mathrm{O}^{+} \mathrm{CD} 21^{\text {high }} \mathrm{CD} 23^{\text {low/ }}-(\mathrm{MZ} \mathrm{B}), \mathrm{B} 22 \mathrm{O}^{+} \mathrm{CD} 21^{\text {int }} \mathrm{CD} 23^{\text {high }}(\mathrm{FO} B)$ in the spleen. Individual fractions defined in (c) were used for calculations. Data show the mean \pm S.E.M. The differences between WT and KO mice were examined for statistical significance that was indicated as asterisks: ${ }^{*} P<0.05 ;{ }^{* \star} P<0.01$; ${ }^{\star \star \star} P<0.001$

after Gpr97 deletion, whereas FO B cells $\left(\mathrm{B} 220^{+}\right.$ $\mathrm{CD} 21^{\text {int }} \mathrm{CD} 23^{\text {high }}$ ) were markedly decreased by 9 -fold in percentage and 10-fold in absolute numbers (Figure $3 \mathrm{~d}$, bottom).

$\mathrm{Gpr}^{-1-}$ mice display abnormal splenic architecture. Microscopic evaluation of the spleen of $\mathrm{Gpr}^{-1-}$ mice showed a disruption of normal architecture characterized by diffuse, irregular follicular (B-cell) areas with an absence of a discrete perifollicular MZ (Figures $4 a$ and b). These morphological abnormalities were substantiated after immunohistochemical detection of $B$ cells (Figures $4 c$ and $d$ ). To corroborate the proportional differences in the mature B-cell populations between $G p r 97^{-/-}$and WT controls, histochemical analysis was performed. Sections were stained with anti-IgM for B cells, and MOMA-1 for metallophilic macrophages to delineate the border between the $M Z$ and the follicular area (Figures $4 e$ and f). Consistent with the increased numbers of MZ B cells observed in flow cytometry, the $\mathrm{MZ}$ area of $\mathrm{Gpr}^{-1-}$ mouse spleen was larger than that of WT controls.

The abnormal architecture of $G p r 97^{-/-}$spleen suggested that immune responses depending on cellular interactions in the follicle might not be fully functional. To test this hypothesis, the germinal centers (GCs) were examined in KO and WT mice after secondary immunization. GCs are typical structures formed in secondary lymphoid organs during the course of a TD immune response, and are the place where affinity maturation, class switching and memory cell formation take place. ${ }^{36}$ Spleen sections were stained with peanut agglutinin (PNA) for the presence of GCs (Figures $4 \mathrm{~g}$ and $\mathrm{h}$ ). The results revealed that PNA-binding GC B cells clearly developed in the spleen of Gpr97-KO mice, which indicated an intact GC formation despite Gpr97 deletion.

Impaired immunoglobulin production, humoral primary and secondary immune responses in $\mathrm{Gpr}^{-1-}$ mice. To determine whether Gpr97 deficiency had an effect on Ig production, Ig titers were assessed in 3-month-old WT and $\mathrm{Gpr}^{-1-}$ mice by ELISA. As shown in Figure 5a, levels of $\lg 1$ 1, IgG2b and IgA were significantly decreased, whereas IgG2a level was significantly increased in $\mathrm{KO}$ mice as compared with WT controls. IgM and IgG3 levels were similar between WT and KO mice. Next, we analyzed the ability of Gpr97-KO mice to mount antibody responses to $\mathrm{TI}$ and TD antigens. At day 14 after immunization with $\mathrm{TI}$ antigen 

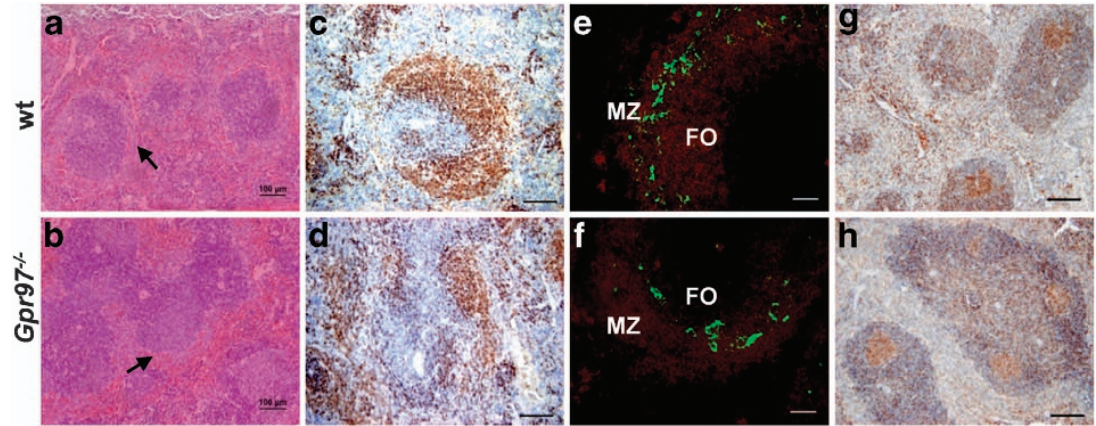

Figure 4 Histopathological and immunohistochemical analysis of the spleen of WT and KO mice ( $n=4$, each group). (a) and (b) Spleen sections from 12-week-old WT and KO mice were stained with H\&E. Perifollicular MZs were indicated by arrows. Scale bar, $100 \mu \mathrm{m}$. (c) and (d) Immunohistochemistry of spleen sections from (a) and (b) were stained with B cell-specific mAb B220. Scale bar, $200 \mu \mathrm{m}$. (e) and (f) Histological section of Gpr97-KO spleen showed an increased population of IgM ${ }^{+}$cells (labeled in red with anti-IgM Ab) in MZ. The green signals represent FITC-labeled anti-MOMA-1, which is specific for metallophilic macrophages that separate the MZ from the follicles. Scale bar, $200 \mu \mathrm{m} .(\mathbf{g})$ and $(\mathbf{h})$ Mice were killed 1 week after secondary immunization with IFA-OVA. Sections of spleen were labeled with PNA (brown). Hematoxylin counterstain was used. Scale bar, $200 \mu \mathrm{m}$
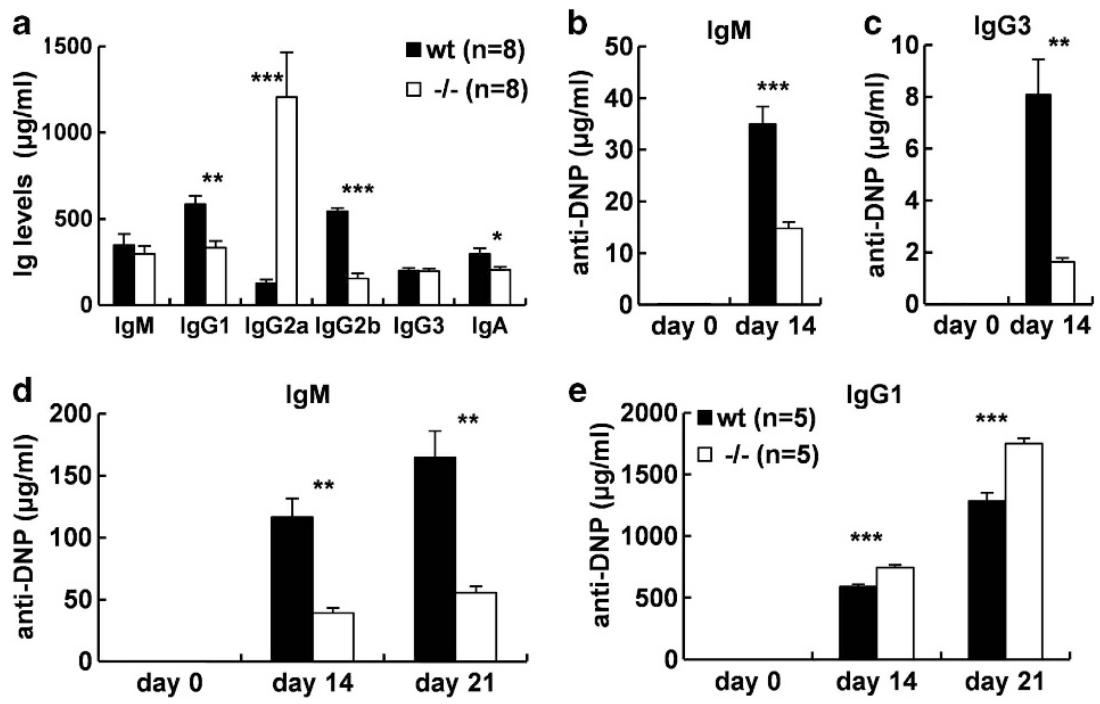

Figure 5 Serum Ig levels, TI and TD antigen responses in WT and KO mice. (a) IgM, lgG1, IgG2a, lgG2b, IgG3 and IgA levels in the sera of 3-month-old WT ( $n=8$ ) and KO $(n=8)$ mice were evaluated by ELISA. Serum levels of $\operatorname{lgM}(\mathbf{b})$ and $\operatorname{lgG} 3(\mathbf{c})$ anti-DNP antibodies were measured before $\left(t_{0}\right)$ and 14 days after $\left(t_{14}\right)$ immunization with the $\mathrm{TI}$ antigen DNP-Ficoll in WT and KO mice. Serum levels of IgM (d) and IgG1 (e) anti-DNP antibodies were measured before $\left(t_{0}\right)$, at the time of secondary immunization $\left(t_{14}\right)$ and 7 days after secondary immunization $\left(t_{21}\right)$ with the TD antigen DNP-KLH in WT and KO mice. Data show the mean \pm S.E.M. The differences between WT and KO mice were examined for statistical significance that was indicated as asterisks: ${ }^{*} P<0.05 ;{ }^{* \star} P<0.01 ;{ }^{* \star} P<0.001$

DNP-Ficoll, production of both IgM and IgG3 anti-DNP antibodies was reduced in Gpr97-KO mice as compared with WT controls (Figures $5 b$ and $c$ ). The amount of DNPspecific IgM antibodies after TD immunization was lower in Gpr97-KO mice than that in controls. Level of DNP-specific IgG1 instead was higher in mutant mice after TD immunization (Figures 5d and e). These data indicate that Gpr97deficient animals are able to make both TD primary and secondary immune responses, consistent with the data of the GC formation in Gpr97-KO mice, as well as TI responses, although at reduced levels compared with WT animals.

Downregulation of lambda 5 expression in Gpr97deficient mice. The observation that abnormal B-cell development in $\mathrm{KO}$ mice prompted us to evaluate the expression pattern of a multitude of different transcriptional factors by real-time PCR, which have been linked to distinct stages of the life of B lymphocytes, such as differentiation in the BM, migration to the peripheral organs and antigeninduced activation. ${ }^{37,38}$ The mRNA expression levels of several genes were found unaltered as compared with those in WT control except for lambda 5 in both BM (Figure 6a) and spleen (Figure 6b). The lambda 5 transcript is reduced by $27.8 \%$ and $84.1 \%$ in BM and spleen of $\mathrm{Gpr}^{-1-}$ mice, respectively. To confirm this observation, we carried out flow cytometric analysis to examine the percentage of lambda 5-positive cells in BM. The results showed that the percentage of lambda 5-positive cells was reduced by

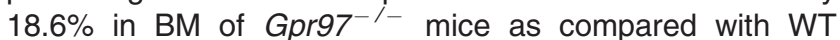
controls, and the difference was statistically significant 

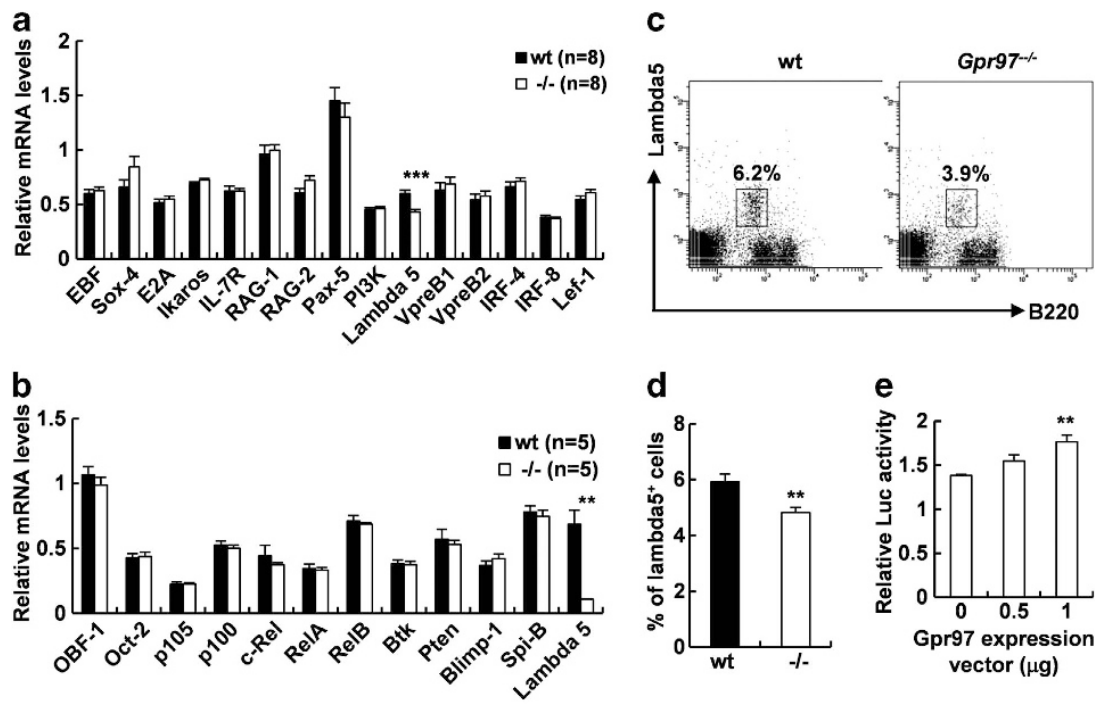

Figure 6 Gpr97 deficiency leads to downregulation of lambda 5. (a) The expression levels of the genes related to B-cell development were evaluated by real-time qPCR in $\mathrm{BM}$ cells. There was a reduction in lambda $5 \mathrm{mRNA}$ level in $\mathrm{KO}$ mice as compared with WT mice ( $n=8$ for each group). (b) Real-time qPCR analysis of the expression levels of genes in splenocytes. Lambda 5 mRNA level was reduced in KO mice as compared with WT mice ( $n=8$ for each group). (c) Staining of BM cells from WT and KO mice ( $n=7$ for each group) with anti-B220 and anti-lambda 5 antibodies. (d) Relative numbers of lambda $5^{+}$cell were presented as mean \pm S.E.M. $(n=7)$. (e) Lambda 5 reporter construct and Gpr97 expression vector were co-transfected into Hela cells. Relative luciferase activity was monitored in triplicate $48 \mathrm{~h}$ post transfection. The differences between two groups were examined for statistical significance that was indicated as asterisks in panels (a), (d) and (e): ${ }^{* \star} P<0.01 ;{ }^{* \star *} P<0.001$

(Figures $6 \mathrm{c}$ and $\mathrm{d}$ ). To further investigate the relationship between Gpr97 and lambda 5, reporter construct with lambda 5 promoter inserted upstream of the luciferase coding region in the pGL3 luciferase reporter vector and Gpr97 expression vector were transiently co-transfected into Hela cells, and results showed that luciferase activity in Hela cells increased with dose of Gpr97 expression vector, as compared with those co-transfected with pcDNA ${ }^{\mathrm{TM}} 3.1 /$ mycHis ( - ) B control vector (Figure 6e). These data suggest that Gpr97 could positively regulate lambda 5 expression at transcriptional level.

\section{A crucial role of Gpr97 in regulating CREB signaling} pathway. Given that Gpr97 is an orphan GPCR, we presumed that Gpr97 would be more likely to regulate lambda 5 indirectly. Through a literature search, we found that Aiolos, a member of the Ikaros family of transcription factors, is required for the efficient silencing of lambda $5 .^{39}$ To determine whether the decreased level of lambda 5 is due to an increased Aiolos level in Gpr97 ${ }^{-/-}$mice, we analyzed the Aiolos level in the spleen by real-time PCR. As expected, an increased Aiolos expression level was observed in the spleen of $\mathrm{KO}$ mice as compared with WT controls (Figure 7a). Then, we analyzed the promoter of Aiolos by TFSEARCH software (Computational Biology Research Center, Tsukuba, Japan) for the purpose of finding potential transcription factor binding sites. As predicted, a CREBbinding site was identified in Aiolos promoter region. The transcription factor CREB is one of the components of many signaling cascades, and is regulated by the GPCRCAMP-PKA pathway. Thus, we examined the levels of pCREB and total CREB in the spleen of $G p r 97^{-/-}$mice by western blot analysis. As shown in Figure 7b, the level of
pCREB in total cell lysate from the spleen of $\mathrm{KO}$ mice was found increased while CREB level remains comparable to that of WT control. Most strikingly, both CREB and pCREB levels in the nuclear extract were found dramatically increased as compared with WT controls, suggesting that Gpr97 could negatively regulate CREB signaling. Thus, we believe that the ablation of Gpr97 leads to increased phosphorylation of CREB, and the increased level of pCREB may enhance the expression level of Aiolos, which downregulates the expression of lambda 5 .

Gpr97 negatively regulates Nf- $\boldsymbol{k} \mathbf{b}$ signaling. BM-derived $B$ cells make an important cell fate choice to develop into either FO B cells or MZ B cells in the spleen, which depends on signaling through $\mathrm{BCR}$, Notch2, the receptor for $\mathrm{B}$ cellactivating factor and the canonical nuclear factor $-\kappa \mathrm{B}(\mathrm{NF}-\kappa \mathrm{B})$ pathway, as well as signals involved in the migration and anatomical retention of $\mathrm{MZ} \mathrm{B}$ cells. ${ }^{40}$ Hence, it is possible that Gpr97 deletion might affect BAFF, NF- $\kappa$ B or Notch2 expression in spleen. This possibility was elucidated by the following three experiments. First, we examined the serum BAFF level by ELISA, and found there is no difference between the two groups (Figure 7c). Second, western blot analysis showed that constitutive p105, p65 and p50 protein levels were increased to some extent in the total cell lysates of $\mathrm{Gpr}^{-1-}$ splenocytes when compared with that of WT control. Accordingly, higher levels of p65 and p50 in the nuclear extracts of $\mathrm{Gpr} 97^{-/-}$splenocytes were observed with comparable levels of p65, p50, as well as p105 proteins in cytoplasmic fraction and responses to LPS stimulation between two groups (Figure 7d). These results indicate that Gpr97 deficiency leads to increased constitutive expression and activation of $\mathrm{Nf}-\kappa \mathrm{b}$ components examined, suggesting 
a
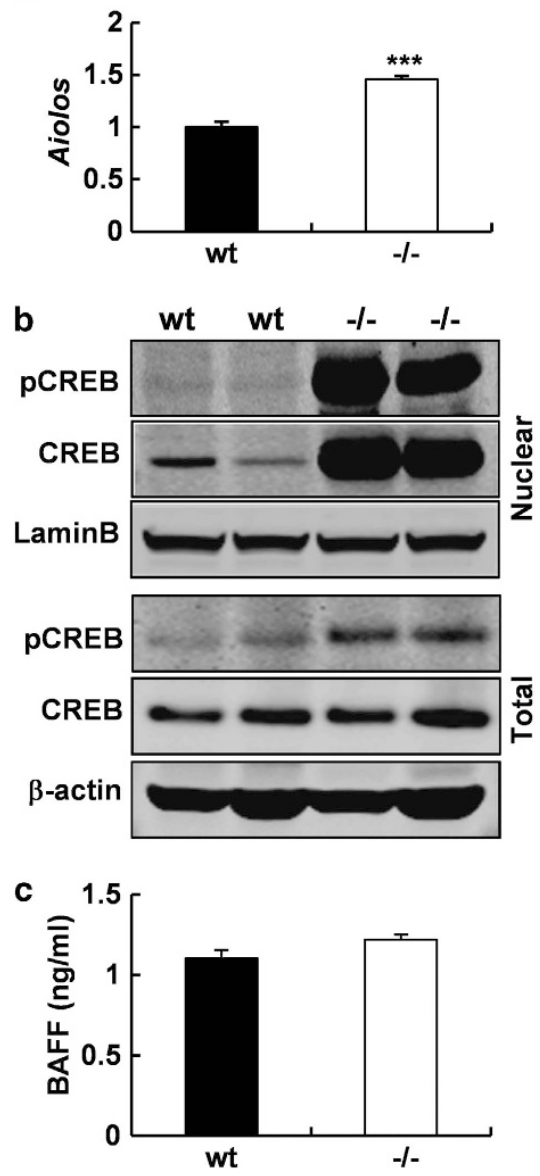

d LPS

$(-)$
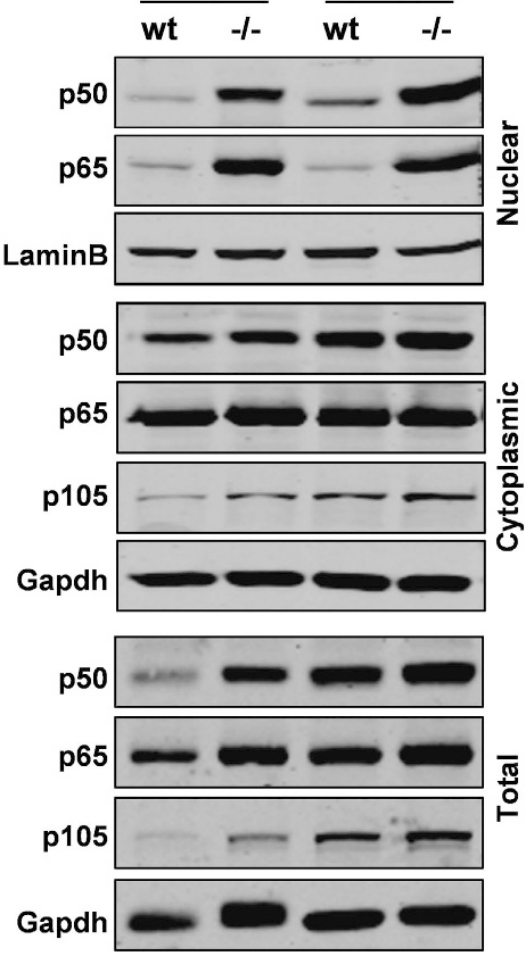

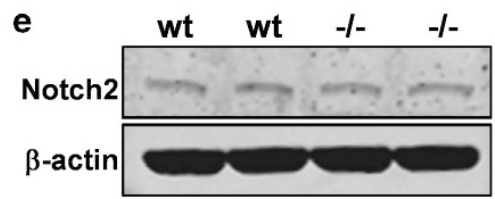

Figure 7 pCREB and Aiolos levels were increased in the spleen of Gpr97-KO mice. (a) Real-time qPCR analysis of Aiolos expression levels in splenocytes $(n=5$ for each group). Data show the mean \pm S.D. (b) Western blot images representing the expressions of pCREB and total CREB in total cell lysates and nuclear fractions from spleen. (c) No difference was observed in serum BAFF level between WT and KO mice $(n=5$, each group). Data show the mean \pm S.E.M. (d) Immunoblots of NF- $\kappa$ B1/p105/p50 and p65 in whole-cell lysates, cytoplasmic and nuclear fractions from splenocytes. (e) Notch2 protein expression was analyzed in spleen by western blot. The differences between WT and KO mice were examined for statistical significance that was indicated as asterisks: ${ }^{\star \star \star} P<0.001$

that Gpr97 may negatively regulate Nf- $\kappa \mathrm{b}$ signaling. Third, the level of Notch2 in the spleen had no significant difference between WT and mutant mice, as demonstrated by western blot analysis (Figure 7e).

\section{Discussion}

A previous study has established a Gpr97-deficient mouse model, which displays no any significant quantitative or qualitative defects in B- and T-cell development. ${ }^{41}$ Such discrepancy may attribute to the different mouse genetic background to that in this study. The mice used in the previous study have a homogenous 129Sv genetic background. However, the mutant mice used in this study are maintained in a mixed 129Sv/C57BL/6 background. Many studies have already addressed that genetic background is of great importance to immune research. ${ }^{42,43}$ Through intensive phenotypic analysis, we have demonstrated that Gpr97 has a crucial role in maintaining normal splenic architecture, regulating B-lymphocyte development, specifically follicular
B-cell development. To understand the molecular events underlying the impaired B-cell development, we have tested the expression levels of multiple transcription factors which have been demonstrated to have a role in B-cell development. For example, E2A and EBF control the immunoglobulin-gene rearrangement, ${ }^{44}$ Blimp1 is induced upon terminal differentiation to plasma cells ${ }^{45}$ and NF- $\kappa$ B is described as a nuclear transactivator of the immunoglobulinlight chain enhancer and a cytokine-inducible transcription factor governing the expression of an important set of genes involved in inflammation and cell survival. ${ }^{37}$ Lambda 5 , a component of pre-BCR, is expressed in B-cell development before conventional $L$ chains. Deficiency of lambda 5 leads to a block in B-cell development in BM at the pre-B-cell stage, resulting in a marked decrease in the number of mature $B$ cells in the periphery. The proportion was increased but not the absolute number of $\mathrm{MZ} B$ cells, whereas FO B cells were decreased. Lambda $5 \mathrm{KO}$ mice are able to mount not only primary but also secondary TD and TI responses, albeit at reduced levels, ${ }^{46,47}$ similar to the phenotypes observed in Gpr97 $^{-/-}$mice. 
To understand the relationship between Gpr97 and lambda 5, we discovered that the expression level of Aiolos which is required for silencing lambda 5, was significantly increased in the spleen of $G p r 97^{-/-}$mice. Interestingly, the promoter of Aiolos has a CREB-binding site predicted by TFSEARCH software. CREB, a cellular transcription factor, is activated by phosphorylation at Ser-133. ${ }^{48}$ The kinase responsible for this activating phosphorylation was identified as cAMPdependent protein kinase, PKA. PKA activity is regulated by molecules that can alter cAMP levels, and hence by GPCRs, which regulate adenylate cyclase activity. Based on the previous findings, we examined the phosphorylation level of CREB in the $\mathrm{Gpr}^{-1-}$ mice and found an increased phosphorylation level of CREB in both nuclear extracts and whole-cell lysates from the spleen of $\mathrm{Gpr} 97^{-1-}$ mice. This observation is in accordance with the previous finding that Gpr97 is coupled to $G_{0}$, which means that the inactivation of Gpr97 would lead to an increase in cAMP levels in target cells. According to these results, it is reasonable that Gpr97 modulates the expression of lambda 5 via Aiolos. We have noted that the follicular type I B-cell population was preserved in Aiolos-deficient mice, in which the strength of BCR signaling was increased, but the number of splenic $M Z B$ cells was markedly decreased, as well as $M Z$ precursor $B$ cells. When the increased BCR signal strength in Aiolos-deficient mice was abrogated by crossing these mice with Xid mice, no less of $\mathrm{MZ}$ or $\mathrm{MZ}$ precursor $\mathrm{B}$ cells was observed, which indicates that increased $B C R$ signaling hampers the differentiation of maturing $\mathrm{B}$ cells into $\mathrm{MZB}$ cells and Aiolos was not directly required for $\mathrm{MZ}$ B-cell development. ${ }^{49}$ Taken together, these observations suggest that an increased Aiolos level may partially contributes to the enhancement of MZ B-cell development in $\mathrm{Gpr} 97^{-}$ mice.

There are several mechanisms that may explain the cell fate determination to develop into either $\mathrm{FO} B$ cells or $\mathrm{MZ} B$ cells in the spleen. First, BCR signaling strength is reported to have a critical role in mature B-cell fate decision. FO B-cell development is dependent on strong $B C R$ signaling, whereas signals driving $M Z$ B-cell development are weak. ${ }^{40}$ Second, the Notch signaling pathway is essential for mature B-cell fate determination. The mice lacking Notch $2,{ }^{50} R b p f^{51}$ or $D I l 1^{52}$ have defects in $\mathrm{MZ}$ B-cell development. Third, BAFF and $\mathrm{NF}-\kappa \mathrm{B}$ are also required for peripheral $\mathrm{B}$ cell development. In IL-7 transgenic mice which have a dramatic increase in the FO B-cell compartment, ${ }^{53} \mathrm{MZ}$ B-cell number was reduced probably due to lower overall levels of BAFF. MZ B-cell development is defective in mice that lack p50. ${ }^{54}$ The absence of Rel and p65 also affects MZ B-cell development partially, which indicates that p50-Rel and p65-Rel complexes might be required for $\mathrm{MZ} B$-cell development. Accordingly, we examined the levels of BAFF, NF- $\kappa \mathrm{B}$ and Notch2 in the spleens of WT and KO mice, and found that nuclear p50 and p65 were markedly increased in Gpr97-null mice, whereas serum BAFF and Notch2 proteins had no differences between WT and $\mathrm{KO}$ mice. These findings suggest that the enhanced Nf- $\kappa \mathrm{b}$ signaling strength may contribute to an increase in $\mathrm{MZ} B$ cells in $\mathrm{Gpr} 7^{-1-}$ mice, whose phenotype is opposite to that of $p 50^{-1-}$ or $p 65^{-1-}$ mice. The question is how Gpr97 affects the levels of nuclear p50 and p65 in splenocytes. A possible inference is that PKA activation is able to simulate PKC and p38 MAPK, leading to IKK-dependent NF- $\kappa$ B activation. ${ }^{55}$
In summary, our study uncovers a novel biological function of Gpr97 in regulating B-cell development, especially CREB and NF- $\kappa$ B signaling pathways, implying Gpr97 as a potential therapeutic target for treatment of immunological disorders.

\section{Materials and Methods}

Mice. The mutant mice were generated by using a homologous recombination method and maintained on a mixed 129Sv/C57BL/6 background and housed under specific pathogen-free conditions at a constant room temperature of $22-24^{\circ} \mathrm{C}$ with a 12-h light/dark cycle. Animal protocols and experiments were approved by the Animal Use and Care Committee of Shanghai Research Center for Model Organisms (Permit Number: 20110007). Age-matched littermates (12-week old) with different genotypes were used for phenotypic analyses. More detailed description on Materials and Methods can be found in the Supplementary Information on line.

Immunization and determination of serum Ig levels. Titers of Ig in serum were tested using ELISA. In brief, 96-well plates were coated with goat antimouse lg capture antibody overnight at $4{ }^{\circ} \mathrm{C}$, followed by incubation with diluted serum samples and developed with HRP-conjugated goat anti-mouse isotypespecific antibodies and substrate ABTS (Southern Biotechnology, Birmingham, AL, USA). Reactions were read at $405 \mathrm{~nm}$ at $10 \mathrm{~min}$ after substrate addition using a microplate reader (BioTek, Winooski, VT, USA). To elicit TI antigen responses, gender-matched 12-week-old WT and KO mice were intraperitoneally injected with $50 \mu \mathrm{g}$ DNP-conjugated Ficoll (Biosearch Technologies, Petaluma, CA, USA). Sera were collected at the time of immunization and 14 days later, and anti-DNP IgM and IgG3 antibody titers were measured by ELISA. For TD antigen responses, 12-week-old WT and KO mice were intraperitoneally injected with $100 \mu \mathrm{g}$ DNPkeyhole limpet hemocyanin (KLH, Biosearch Technologies) with CFA. After 2 weeks, mice were injected again with $10 \mu \mathrm{g}$ DNP- KLH with IFA. Sera were collected on the day of the primary immunization, at the time of the secondary immunization, and another week later. Antibodies to DNP were measured by ELISA using plates coated with DNP-BSA (Biosearch Technologies).

Cell culture. Suspensions of splenocytes (after isotonic erythrocyte lysis) were cultured in complete RPMI 1640 medium containing 10\% FBS, 1\% HEPES, $1 \%$ L-glutamate, $1 \%$ pen/strep and $0.1 \% 2$-mercapthoethanol with or without LPS $(20 \mu \mathrm{g} / \mathrm{ml})$ for 24 or $48 \mathrm{~h}$. Monitoring of apoptosis or necrosis was performed by staining with annexin V and PI (Invitrogen, Grand Island, NY, USA) according to the manufacturer's instructions. Cells negative for PI but positive for annexin V were defined as apoptotic cells. Late apoptotic and necrotic cells were identified by strong PI staining.

Histological analysis. Spleens were fixed for $24 \mathrm{~h}$ in $10 \%$ neutral buffered formalin, embedded in paraffin, sectioned $(5 \mu \mathrm{m})$ and then stained with hematoxylin and eosin. For immunohistochemistry, splenic sections were incubated with the antibodies against B220 (BD Pharmingen, Franklin Lakes, $\mathrm{NJ}$, USA) in blocking buffer overnight at $4^{\circ} \mathrm{C}$. After washing, the sections were stained with biotin-labeled rat second antibody (KPL), and label developed using an ABC kit (Vector Laboratories, Burlingame, CA, USA). For immunofluorescence, spleens were frozen in Tissue-Tek OCT compound (Sakura, Torrance, CA, USA) and sectioned at a thickness of $8 \mu \mathrm{m}$. Sections were then fixed for $15 \mathrm{~min}$ in $4 \%$ paraformaldehyde (PFA) and stored at $-20{ }^{\circ} \mathrm{C}$. For labeling, slides were washed, incubated for $10 \mathrm{~min}$ in PBS containing $0.25 \%$ Triton X-100 and blocked for $30 \mathrm{~min}$ in $1 \%$ BSA in PBS. Then sections were labeled overnight at $4{ }^{\circ} \mathrm{C}$ with primary antibody in blocking buffer. After extensive washing in PBS, slides were mounted in Antifade Mounting Medium (Beyotime, Shanghai, China). Primary antibodies used: FITC-MOMA-1 (AbD Serotec, Oxford, UK) and Texas Red-IgM (Vector Laboratories). GC formation in spleen was examined by histological analysis. Sections were labeled with PNA (Vector Laboratories), and color was developed using an $\mathrm{ABC}$ kit (Vector Laboratories).

Analyses of mRNA expression. Total RNA was prepared from different mouse tissues using Trizol Reagent (Invitrogen) according to manufacturer's instructions. mRNA expression levels were assayed by semi- or real-time quantitative RT-PCR. The expression level of $\beta$-actin was used as an endogenous control. Real-time PCR analysis was performed using SYBR Premix Ex Taq kit (Takara, Dalian, China) on an Eppendorf Mastercycler system according to the manufacturer's protocol. All samples were tested in triplicate, and the results were normalized to $\beta$-actin expression. 
Flow cytometry. Single-cell suspensions of BM cells, splenocytes or PB cells were stained using standard procedures. Cells were adjusted to $2 \times 10^{7} \mathrm{cell} / \mathrm{s} / \mathrm{ml}$ in PBS with $2 \%$ FBS and $0.1 \%$ sodium azide, and $1 \times 10^{6}$ cells were incubated $30 \mathrm{~min}$ on ice with appropriately diluted antibodies $(1 \mathrm{ng} / \mu \mathrm{l})$ in a total volume of $100 \mu \mathrm{l}$. After washing three times, the stained cells were analyzed using a flow cytometer (BD FACSAria, BD Pharmingen). Processed samples were analyzed using CellQuest software (BD Biosciences).

Western blot analysis. Whole-cell lysates from BM cells $\left(1-2 \times 10^{7}\right)$ were extracted with RIPA lysis buffer (Thermo Scientific, Waltham, MA, USA). Nuclear protein extracts from spleen were prepared according to the handbook of NE-PER Nuclear and Cytoplasmic Extraction Reagents (Thermo Scientific). The protein concentration was determined using the BCA Protein Assay Kit (Pierce, Rockford, IL, USA). Equal amounts of protein $(60-80 \mu \mathrm{g})$ were separated and transferred onto polyvinylidene fluoride membrane by semi-dry blotting. The membranes were incubated overnight with the antibodies listed in Supplementary Information. Infrared fluorescence on membranes was detected using an Odyssey infrared imaging system (LI-COR). For quantitative determinations, a densitometric analysis of signal bands was performed using Gel-Pro Analyzer software (Media Cybernetics. Bethesda, MD, USA).

Statistical analysis. Quantitative results were presented graphically as mean \pm S.E. The statistical differences in the observed data were compared by one-way ANOVA (analysis of variance). $P<0.05$ was considered statistically significant.

\section{Conflict of Interest}

The authors declare no conflict of interest.

Acknowledgements. This work was partially supported by the Grants from Ministry of Science and Technology of China (2006BAI23B02 and 2011BAl15B02), the Science and Technology Commission of Shanghai Municipality (11DZ2292400 12ZR1421100, 13140901400 and 13DZ2280600), and the E-Institutes of Shanghai Municipal Education Commission (E03003).

1. Pridans C, Holmes ML, Polli M, Wettenhall JM, Dakic A, Corcoran LM et al. Identification of Pax5 target genes in early B cell differentiation. J Immunol 2008; 180: 1719-1728.

2. Lam QL, Lo CK, Zheng BJ, Ko KH, Osmond DG, Wu GE et al. Impaired V(D)J recombination and increased apoptosis among $B$ cell precursors in the bone marrow of c-Abl-deficient mice. Int Immunol 2007; 19: 267-276.

3. Ohashi PS, DeFranco AL. Making and breaking tolerance. Curr Opin Immunol 2002; 14 744-759.

4. Shapiro-Shelef M, Calame K. Regulation of plasma-cell development. Nat Rev Immunol 2005; 5: 230-242.

5. Pillai S, Cariappa A, Moran ST. Marginal zone B cells. Annu Rev Immunol 2005; 23 161-196.

6. Gururajan M, Simmons A, Dasu T, Spear BT, Calulot C, Robertson DA et al. Early growth response genes regulate $B$ cell development, proliferation, and immune response J Immunol 2008; 181: 4590-4602.

7. Cariappa A, Tang M, Parng C, Nebelitskiy E, Carroll M, Georgopoulos K et al. The follicular versus marginal zone $B$ lymphocyte cell fate decision is regulated by Aiolos, Btk, and CD21. Immunity 2001; 14: 603-615.

8. Cariappa A, Liou HC, Horwitz BH, Pillai S. Nuclear factor kappa B is required for the development of marginal zone B lymphocytes. J Exp Med 2000; 192: 1175-1182.

9. Weih DS, Yilmaz ZB, Weih F. Essential role of RelB in germinal center and marginal zone formation and proper expression of homing chemokines. J Immunol 2001; 167 $1909-1919$.

10. Quong MW, Martensson A, Langerak AW, Rivera RR, Nemazee D, Murre C. Receptor editing and marginal zone $B$ cell development are regulated by the helix-loop-helix protein E2A. J Exp Med 2004; 199: 1101-1112.

11. Guo F, Weih D, Meier E, Weih F. Constitutive alternative NF-kappaB signaling promotes marginal zone B-cell development but disrupts the marginal sinus and induces HEV-lik structures in the spleen. Blood 2007; 110: 2381-2389.

12. Yona S, Lin HH, Siu WO, Gordon S, Stacey M. Adhesion-GPCRs: emerging roles for novel receptors. Trends Biochem Sci 2008; 33: 491-500.

13. Harmar AJ. Family-B G-protein-coupled receptors. Genome Biol 2001; 2, REVIEWS 3013.1-REVIEWS 3013.10.

14. Foord SM, Jupe S, Holbrook J. Bioinformatics and type II G-protein-coupled receptors. Biochem Soc Trans 2002; 30: 473-479.
15. Lin HH, Chang GW, Davies JQ, Stacey M, Harris J, Gordon S. Autocatalytic cleavage of the EMR2 receptor occurs at a conserved $G$ protein-coupled receptor proteolytic site motif. J Biol Chem 2004; 279: 31823-31832.

16. Shima $Y$, Kengaku M, Hirano $T$, Takeichi M, Uemura T. Regulation of dendritic maintenance and growth by a mammalian 7-pass transmembrane cadherin. Dev Cell 2004; 7: 205-216.

17. Tissir F, Qu Y, Montcouquiol M, Zhou L, Komatsu K, Shi D et al. Lack of cadherins Celsr2 and Celsr3 impairs ependymal ciliogenesis, leading to fatal hydrocephalus. Nat Neurosci 2010; 13: 700-707.

18. Langenhan T, Promel S, Mestek L, Esmaeili B, Waller-Evans H, Hennig C et al. Latrophilin signaling links anterior-posterior tissue polarity and oriented cell divisions in the $\mathrm{C}$. elegans embryo. Dev Cell 2009; 17: 494-504.

19. Wang T, Ward Y, Tian L, Lake R, Guedez L, Stetler-Stevenson WG et al. CD97, an adhesion receptor on inflammatory cells, stimulates angiogenesis through binding integrin counterreceptors on endothelial cells. Blood 2005; 105: 2836-2844.

20. Hoek RM, de Launay D, Kop EN, Yilmaz-Elis AS, Lin F, Reedquist KA et al. Deletion of either CD55 or CD97 ameliorates arthritis in mouse models. Arthritis Rheum 2010; 62 : 1036-1042.

21. Hamann J, Koning N, Pouwels W, Ulfman LH, van Eijk M, Stacey M et al. EMR1, the human homolog of F4/80, is an eosinophil-specific receptor. Eur J Immunol 2007; 37: 2797-2802.

22. Yona $S$, Lin $H H$, Dri $P$, Davies JQ, Hayhoe RP, Lewis $S M$ et al. Ligation of the adhesion-GPCR EMR2 regulates human neutrophil function. FASEB J 2008; 22: 741-751.

23. Matmati M, Pouwels W, van Bruggen R, Jansen M, Hoek RM, Verhoeven AJ et al. The human EGF-TM7 receptor EMR3 is a marker for mature granulocytes. J Leukoc Biol 2007; 81: $440-448$.

24. Vallon M, Essler M. Proteolytically processed soluble tumor endothelial marker (TEM) 5 mediates endothelial cell survival during angiogenesis by linking integrin alpha(v)beta3 to glycosaminoglycans. J Biol Chem 2006; 281: 34179-34188.

25. Waller-Evans H, Promel S, Langenhan T, Dixon J, Zahn D, Colledge WH et al. The orphan adhesion-GPCR GPR126 is required for embryonic development in the mouse. PLOS One 2010; 5: e14047.

26. Fredriksson R, Lagerstrom MC, Hoglund PJ, Schioth HB. Novel human G protein-coupled receptors with long $\mathrm{N}$-terminals containing GPS domains and Ser/Thr-rich regions. FEBS Lett 2002; 531: 407-414.

27. Shashidhar S, Lorente G, Nagavarapu U, Nelson A, Kuo J, Cummins J et al. GPR56 is a GPCR that is overexpressed in gliomas and functions in tumor cell adhesion. Oncogene 2005; 24: 1673-1682.

28. Peng YM, van de Garde MD, Cheng KF, Baars PA, Remmerswaal EB, van Lier RA et al. Specific expression of GPR56 by human cytotoxic lymphocytes. J Leukoc Biol 2011; 90: 735-740.

29. Gupte J, Swaminath G, Danao J, Tian H, Li Y, Wu X. Signaling property study of adhesion G-protein-coupled receptors. FEBS Lett 2012; 586: 1214-1219.

30. Loder F, Mutschler B, Ray RJ, Paige CJ, Sideras P, Torres R et al. B cell development in the spleen takes place in discrete steps and is determined by the quality of $B$ cell receptorderived signals. J Exp Med 1999; 190: 75-89.

31. Vu TT, Gatto D, Turner V, Funnell AP, Mak KS, Norton LJ et al. Impaired B cell development in the absence of Kruppel-like factor 3. J Immunol 2011; 187: 5032-5042.

32. Samanta DN, Palmetshofer A, Marinkovic D, Wirth T, Serfling E, Nitschke L. B cell hyperresponsiveness and expansion of mature follicular B cells but not of marginal zone $\mathrm{B}$ cells in NFATc2/c3 double-deficient mice. J Immunol 2005; 174: 4797-4802.

33. Kanayama N, Cascalho M, Ohmori H. Analysis of marginal zone B cell development in the mouse with limited $B$ cell diversity: role of the antigen receptor signals in the recruitment of B cells to the marginal zone. J Immunol 2005; 174: $1438-1445$

34. Nijnik A, Dawson S, Crockford TL, Woodbine L, Visetnoi S, Bennett $S$ et al. Impaired lymphocyte development and antibody class switching and increased malignancy in a murine model of DNA ligase IV syndrome. J Clin Invest 2009; 119: 1696-1705.

35. Katagiri K, Katakai T, Ebisuno Y, Ueda Y, Okada T, Kinashi T. Mst1 controls lymphocyte trafficking and interstitial motility within lymph nodes. EMBO J 2009; 28: 1319-1331.

36. Thorbecke GJ, Amin AR, Tsiagbe VK. Biology of germinal centers in lymphoid tissue. FASEB J 1994; 8: 832-840.

37. Bartholdy B, Matthias P. Transcriptional control of B cell development and function. Gene 2004; $327:$ 1-23.

38. Schebesta M, Heavey B, Busslinger M. Transcriptional control of B-cell development. Curr Opin Immunol 2002; 14: 216-223.

39. Ma S, Pathak S, Mandal M, Trinh L, Clark MR, Lu R. Ikaros and Aiolos inhibit pre-B-cell proliferation by directly suppressing c-Myc expression. Mol Cell Biol 2010; 30: 4149-4158.

40. Pillai S, Cariappa A. The follicular versus marginal zone B lymphocyte cell fate decision. Nat Rev Immunol 2009: 9: 767-777.

41. Sleckman BP, Khan WN, Xu W, Bassing CH, Malynn BA, Copeland NG et al. Cloning and functional characterization of the early-lymphocyte-specific Pb99 gene. Mol Cell Biol 2000; 20: $4405-4410$. 
42. Gatien ML, Hotchkiss AK, Neigh GN, Dhabhar FS, Nelson RJ. Immune and stress responses in $\mathrm{C} 57 \mathrm{BL} / 6$ and $\mathrm{C} 3 \mathrm{H} / \mathrm{HeN}$ mouse strains following photoperiod manipulation. Neuro Endocrinol Lett 2004; 25: 267-272.

43. Brown DE, Libby SJ, Moreland SM, McCoy MW, Brabb T, Stepanek A et al. Salmonella enterica causes more severe inflammatory disease in C57/BL6 Nramp1G169 mice than Sv129S6 mice. Vet Pathol 2013; 50: 867-876.

44. Romanow WJ, Langerak AW, Goebel P, Wolvers-Tettero IL, van Dongen JJ, Feeney $\mathrm{AJ}$ et al. E2A and EBF act in synergy with the $\mathrm{V}(\mathrm{D}) \mathrm{J}$ recombinase to generate a diverse immunoglobulin repertoire in nonlymphoid cells. Mol Cell 2000; 5: 343-353.

45. Angelin-Duclos C, Cattoretti G, Lin KI, Calame K. Commitment of B lymphocytes to a plasma cell fate is associated with Blimp-1 expression in vivo. J Immunol 2000; 165: 5462-5471.

46. Kitamura D, Kudo A, Schaal S, Muller W, Melchers F, Rajewsky K. A critical role of lambda 5 protein in B cell development. Cell 1992; 69: 823-831.

47. Harfst E, Andersson J, Grawunder U, Ceredig R, Rolink AG. Homeostatic and functional analysis of mature B cells in lambda5-deficient mice. Immunol Lett 2005; 101: 173-184.

48. Gonzalez GA, Yamamoto KK, Fischer WH, Karr D, Menzel P, Biggs W 3rd et al. A cluster of phosphorylation sites on the cyclic AMP-regulated nuclear factor CREB predicted by its sequence. Nature 1989; 337: 749-752.

49. Feng J, Wang H, Shin DM, Masiuk M, Qi CF, Morse HC 3rd. IFN regulatory factor 8 restricts the size of the marginal zone and follicular B cell pools. J Immunol 2011; 186: 1458-1466.

50. Saito T, Chiba S, Ichikawa M, Kunisato A, Asai T, Shimizu K et al. Notch2 is preferentially expressed in mature $B$ cells and indispensable for marginal zone $B$ lineage development. Immunity 2003; 18: 675-685.
51. Tanigaki K, Han H, Yamamoto N, Tashiro K, Ikegawa M, Kuroda K et al. Notch-RBP-J signaling is involved in cell fate determination of marginal zone B cells. Nat Immunol 2002; 3: 443-450.

52. Hozumi K, Negishi N, Suzuki D, Abe N, Sotomaru Y, Tamaoki $\mathrm{N}$ et al. Delta-like 1 is necessary for the generation of marginal zone $B$ cells but not $\mathrm{T}$ cells in vivo. Nat Immunol 2004; 5: 638-644.

53. Ceredig R, Bosco N, Maye PN, Andersson J, Rolink A. In interleukin-7-transgenic mice, increasing $B$ lymphopoiesis increases follicular but not marginal zone B cell numbers. Eur $J$ Immunol 2003; 33: 2567-2576.

54. Moran ST, Cariappa A, Liu H, Muir B, Sgroi D, Boboila C et al. Synergism between NF-kappa B1/p50 and Notch2 during the development of marginal zone B lymphocytes. J Immunol 2007; 179: 195-200.

55. Chio CC, Chang YH, Hsu YW, Chi KH, Lin WW. PKA-dependent activation of PKC, p38 MAPK and IKK in macrophage: implication in the induction of inducible nitric oxide synthase and interleukin-6 by dibutyryl cAMP. Cell Signal 2004; 16: 565-575.

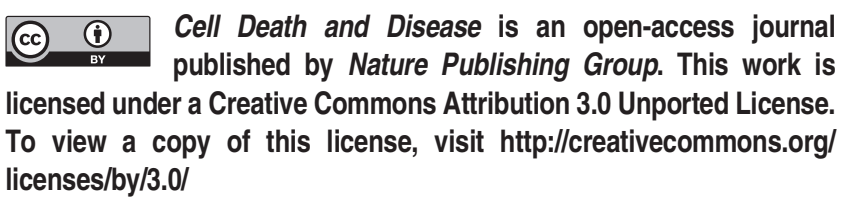

Supplementary Information accompanies this paper on Cell Death and Disease website (http://www.nature.com/cddis) 